\title{
Grüne Blüten
}

\author{
Hilke Steinecke
}

\begin{abstract}
Green is a rather rare flower colour. Four tropical species with green flowers are presented. They can be found in the Tropicarium.

\section{Zusammenfassung}

Grün ist eine eher seltene Blütenfarbe. Es werden vier tropische Arten mit grünen Blüten kurz vorgestellt, die in den Schauhäusern des Tropicariums zu finden sind.
\end{abstract}

\section{Grün ist meine Farbe}

$\mathrm{Zu}$ den häufigen Blütenfarben gehören Gelb, Rot, Orange, Weiß oder Blauviolett. Grüntöne sind bei Blüten vergleichsweise selten. Grüne Blüten heben sich nicht so gut vom grünen Laub ab und grün blühende Arten locken ihre Bestäuber durch einen intensiven Duft an. Grünlich blühende Pflanzenarten werden oft von Käfern, Aasfliegen oder Fledermäusen bestäubt. Eine Auswahl grün blühender Pflanzen ist im Palmengarten auch im Tropicarium zu finden.

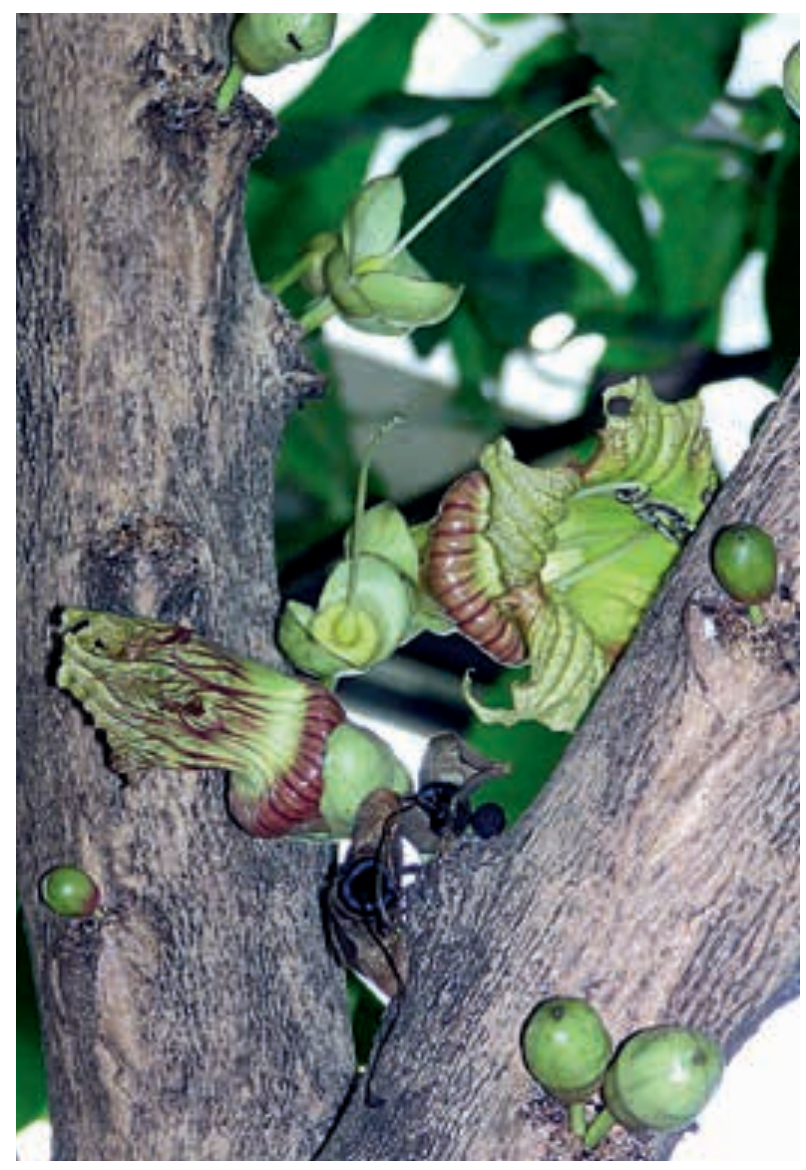

Abb. 1: Blüten von Crescentia cujete.

\section{Crescentia cujete}

Der Kalebassenbaum, ein Vertreter der Bignoniaceae, wird vielerorts in den Tropen kultiviert. Seine etwa $5 \mathrm{~cm}$ großen, grünlich-bräunlichen Blüten sitzen direkt am Stamm oder an älteren Zweigen (Kauliflorie). Sie blühen nur kurz, strömen einen etwas muffigen Duft aus und werden von Fledermäusen bestäubt. Aus den Blüten entwickeln sich auch ohne Bestäubung bis $40 \mathrm{~cm}$ große, kugelige oder elliptische, sehr hartschalige Früchte. Aus diesen stellten die Ureinwohner Amerikas bereits in vorkolumbianischer Zeit diverse Gefäße her. Durch künstliche Einschnürungen der wachsenden Frucht lässt sich die spätere Form beeinflussen, so auch bei den Rumba-Rasseln, durch die die Kalebasse bei uns bekannt wurde. Heute werden viele kunsthandwerkliche Produkte sowie Musikinstrumente nicht mehr vorwiegend aus den Früchten von Crescentia, sondern meist von Lagenaria (Flaschenkürbis) hergestellt. Der Kalebassenbaum ist im Tropicarium sowohl im Tieflandregenwälder-Haus als auch im Savannenund Trockenwälder-Haus zu sehen, wo er über das ganze Jahr hinweg Früchte trägt.

\section{Deherainia smaragdina}

Der Smaragdstrauch ist ein holziger Verwandter der Primeln. Die Gattung Deherainia wurde nach dem im 19. Jahrhundert lebenden französischen Botaniker Pierre Paul Deherain benannt. Sie umfasst die drei Arten D. lageniformis, D. matudae und D. smaragdina. Der Smaragdstrauch kommt von Ost-Mexiko bis Costa Rica vor. Seine smaragdgrünen Blüten fallen zwischen den Blättern kaum auf. Durch den muffigen Geruch der Blüten werden die bestäubenden Fliegen angelockt. Von der 


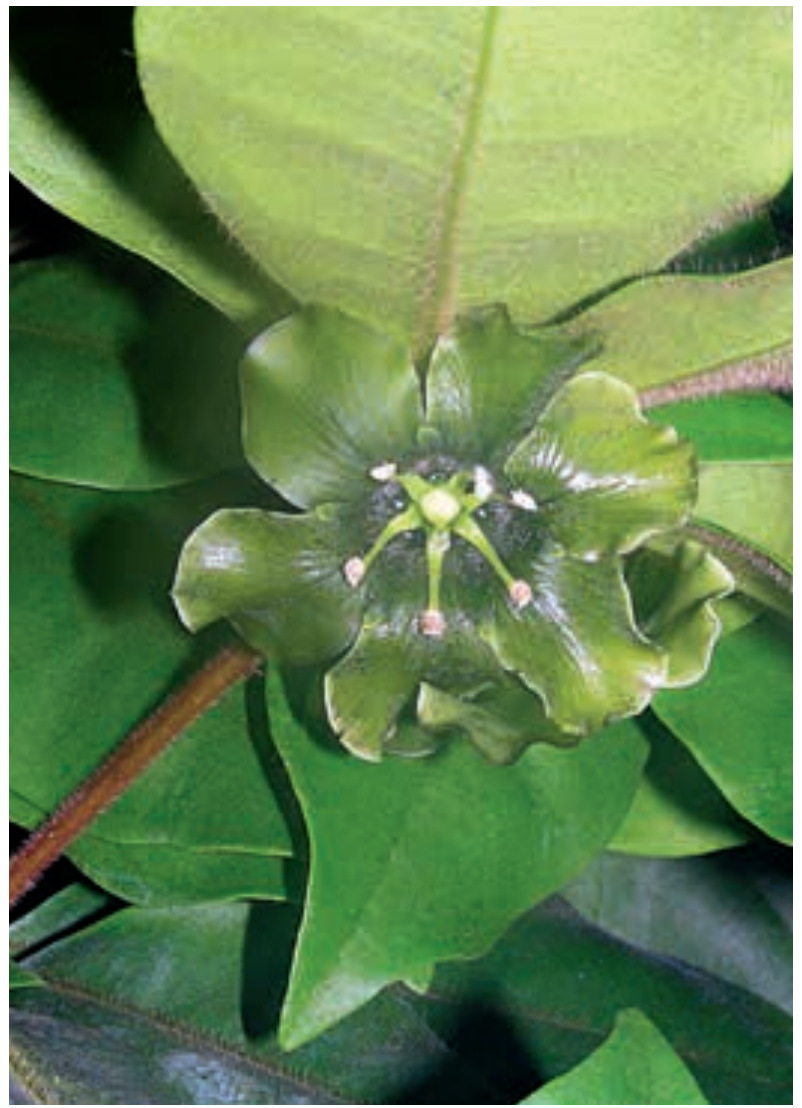

Abb. 2: Deherainia smaragdina. Weibliche Phase der Blüte, die Narbe ist zugänglich.

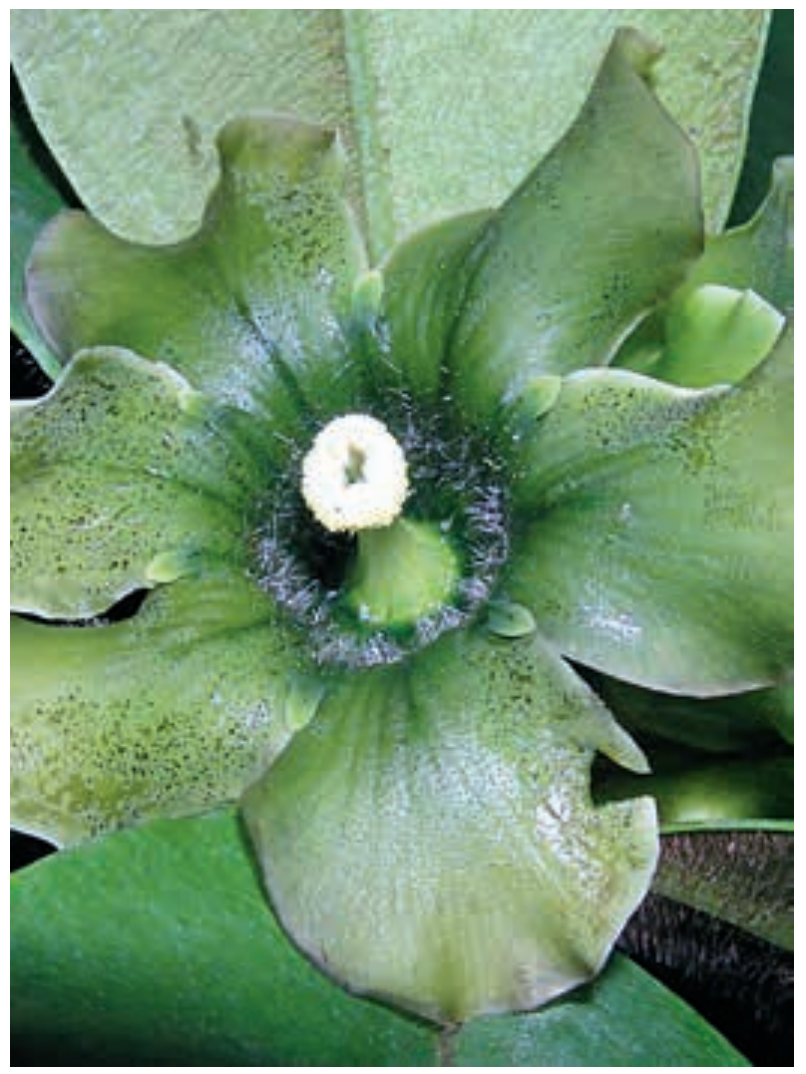

Abb. 3: Deherainia smaragdina. Männliche Phase der Blüte, die Staubblätter sind zum Zentrum geneigt.

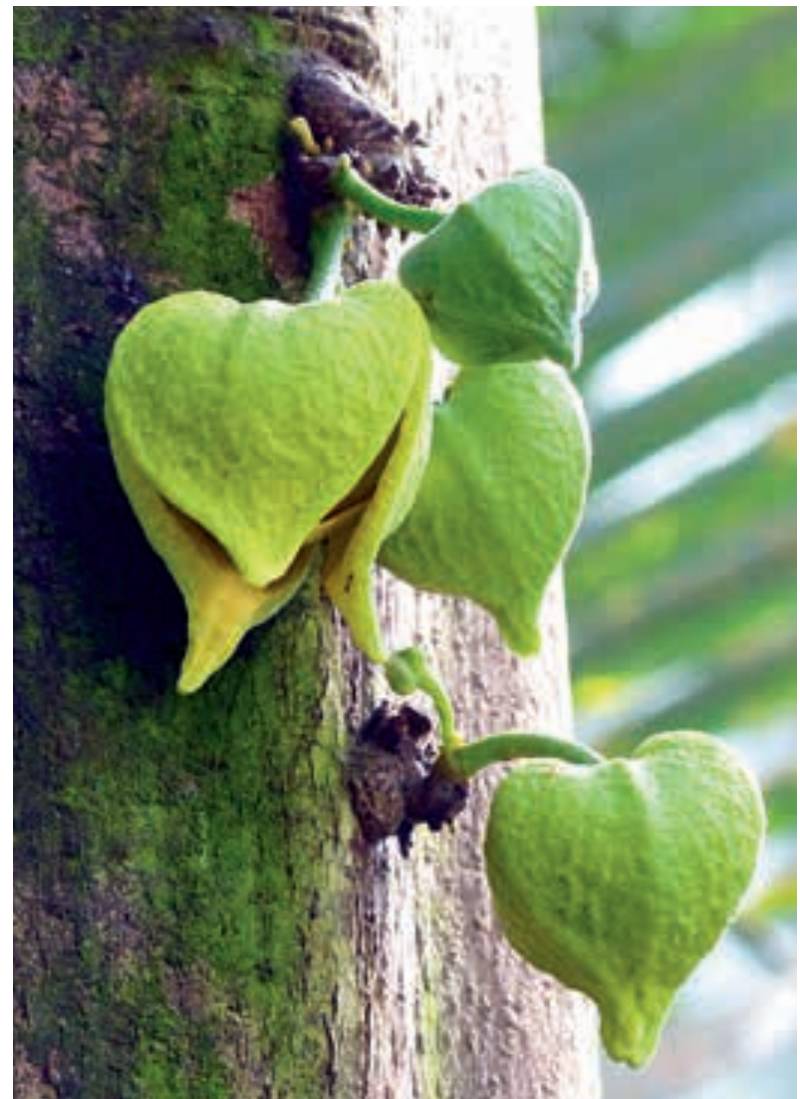

Abb. 4: Die Blüten von Annona muricata befinden sich direkt am Stamm, ein Beispiel für Kauliflorie.

grünen Farbe der Blütenblätter heben sich die blassgelben Narben und Staubblätter ab. Die fünf Staubblätter liegen zunächst der Narbe eng an und biegen sich später nach außen. Der Strauch steht im Monsun- und Passatwälder-Haus.

\section{Annona muricata}

Der Schuppenapfel (auch Sauersack oder Stachelannone genannt) gehört zur artenreichen Familie der Schuppenapfelgewächse (Annonaceae), die heute weltweit vor allem in tropischen Gebieten vorkommen. Der aus Südamerika stammende Schuppenapfel ist eng verwandt mit Cherimoya (Annona cherimola), dessen apfelgroße Früchte seit einiger Zeit auch bei uns angeboten werden. Die gelblichgrünen Blüten von Annona muricata entwickeln sich direkt am Stamm oder an kräftigen Zweigen. Die Blütenblätter sind lederig derb und öffnen sich nicht vollständig. Ähnlich wie bei den Aronstabgewächsen erhitzten sich die Blüten nachts und verströmen einen Duft nach überreifem Obst. Als Bestäuber werden kleinere Käfer angelockt. Die außen stacheligen, grünen 


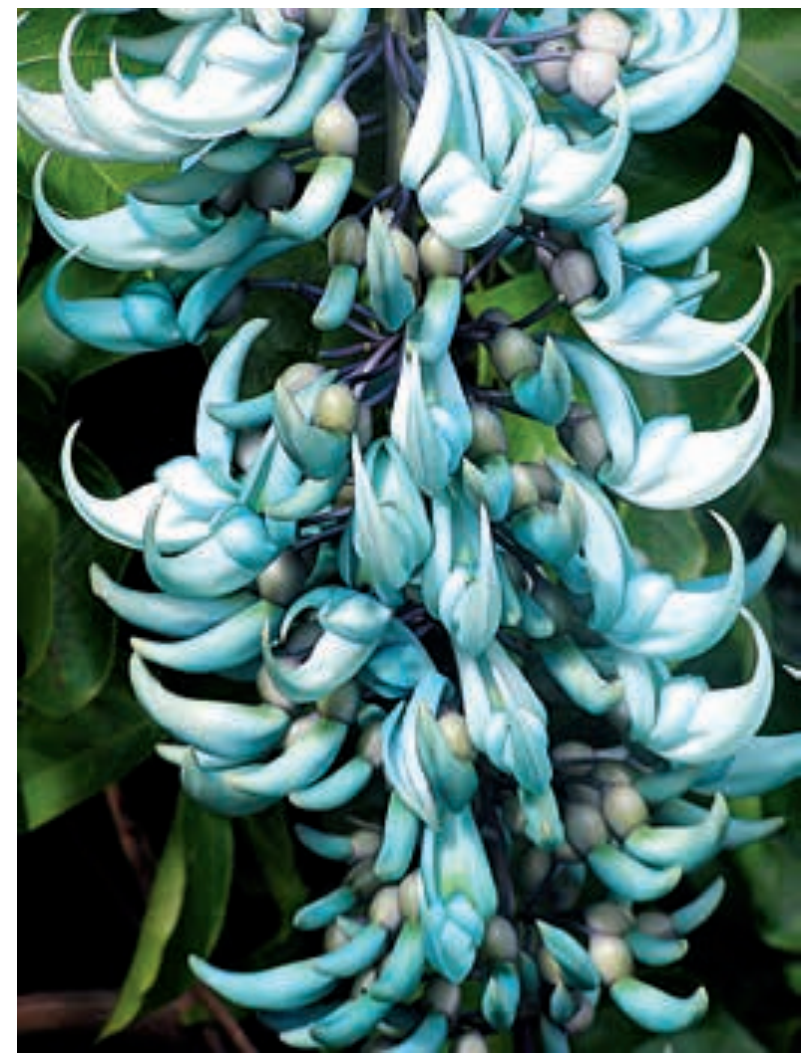

Abb. 6: Die türkisgrünen Blüten von Strongylodon macrobotrys sind schiffchenförmig.

Früchte werden bis $40 \mathrm{~cm}$ lang und $4 \mathrm{~kg}$ schwer. Während der Fruchtentwicklung verwachsen die zunächst freien Fruchtblätter miteinander, so dass eine Sammelbeere entsteht. Die Zahl der Samen entspricht der Anzahl der am Fruchtbau beteiligten Fruchtblätter. Da die Früchte schnell verderben, werden sie nur selten exportiert. Das saftig-saure Fruchtfleisch wird zu Püree verarbeitet oder zur Gewinnung von Saft ausgepresst. Das Palmengarten-Exemplar blüht regelmäßig, die Früchte reifen aber nicht komplett aus. Der große Baum wächst ebenfalls im Monsun- und Passatwälder-Haus.

\section{Strongylodon macrobotrys}

Die Jaderanke ist ein Schmetterlingsblütler ( $\mathrm{Fa}$ baceae). Die lianenähnliche Kletterpflanze ist in den philippinischen Urwäldern heimisch und wird dort als Sabawil bezeichnet. Lianen müssen sehr zugfest und biegsam sein. Bei der Jaderanke sind einige der Triebe wie ein Seil miteinander verdreht. Sie kann bis zu 18 m hoch klettern. Ihre großen Blüten haben eine ungewöhnliche türkisgrüne Farbe. Sie hängen in bis zu $1 \mathrm{~m}$ lan-

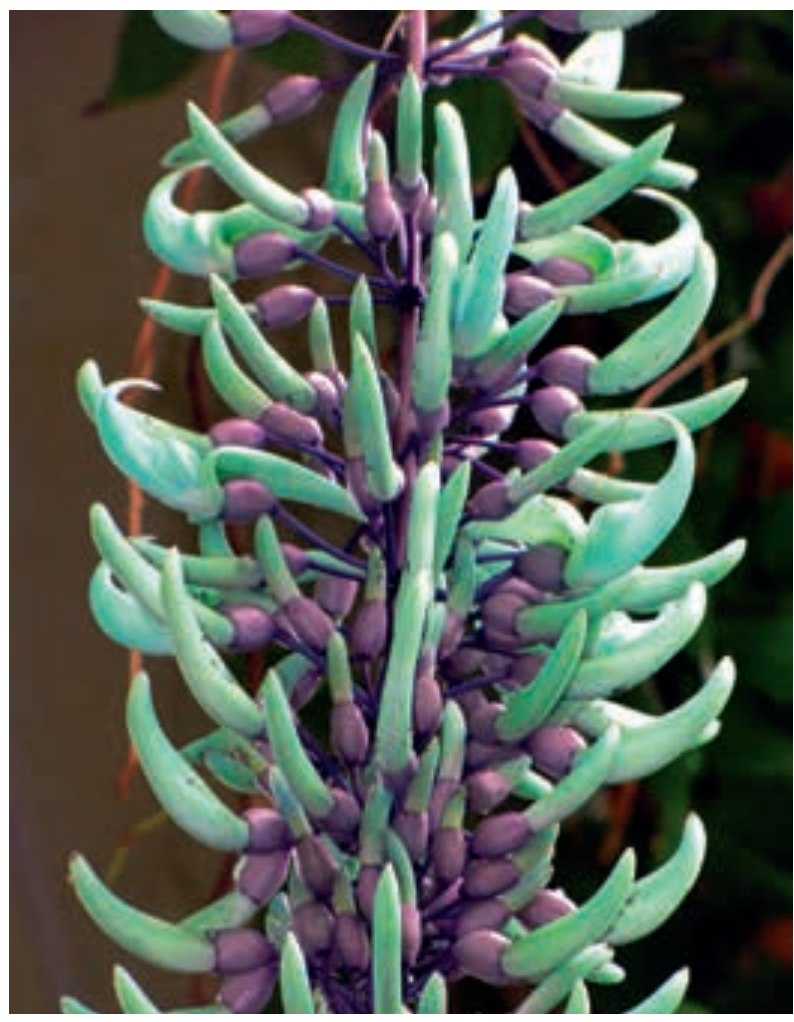

Abb. 7: Knospiger Blütenstand. Hier kontrastieren die violetten Kelchblätter sehr schön mit dem Türkisgrün der Kronblätter.

gen traubigen Blütenständen und haben eine kahnähnliche, etwas nach oben gebogene Form. Sie werden von Fledermäusen bestäubt. Die Art wurde 1937 entdeckt und erst 1953 kultiviert. In tropischen Gebieten ist die Jaderanke wegen ihrer schönen Blüten eine beliebte Gartenpflanze. Im Palmengarten blüht sie selten und unregelmäßig im Tieflandsregenwälder-Haus.

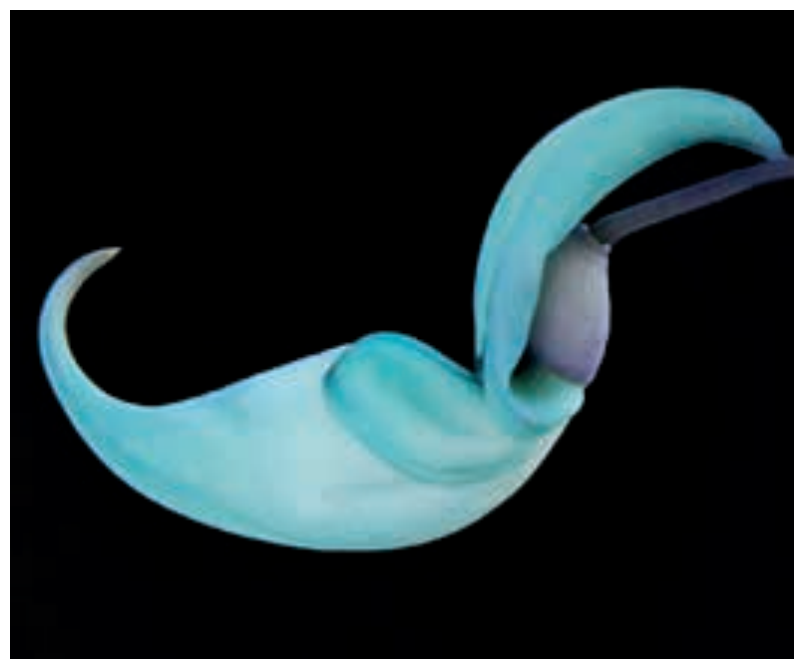

Abb. 8: Einzelblüte von Strongylodon macrobotrys. 